\title{
User-friendly Interface for Electrical Cell-substrate Impedance Spectroscopy System
}

\author{
Jong-Dae Kim, ${ }^{1,2}$ Chan-Young Park, ${ }^{1,2}$ Sang-Oh Park, ${ }^{2,3}$ Hye-Jeong Song, ${ }^{1,2}$ \\ Yu-Seop Kim, ${ }^{1,2}$ Won-Kyun Im, ${ }^{4,5}$ Sung-Ho Jeon, ${ }^{4,5}$ and Jongwon Kim ${ }^{6 *}$ \\ ${ }^{1}$ Department of Convergence Software, Hallym University, \\ 1, Hallymdaehak-gil, Chuncheon-si, Gangwon-do 24252, Korea \\ ${ }^{2}$ Bio-IT Research Center, Hallym University, \\ 1, Hallymdaehak-gil, Chuncheon-si, Gangwon-do 24252, Korea \\ ${ }^{3}$ Department of Computer Engineering, Hallym University, \\ 1, Hallymdaehak-gil, Chuncheon-si, Gangwon-do 24252, Korea \\ ${ }^{4}$ Department of Life Science, Hallym University, \\ 1, Hallymdaehak-gil, Chuncheon-si, Gangwon-do 24252, Korea \\ ${ }^{5}$ Multidisciplinary Genome Institute, Hallym University, \\ 1, Hallymdaehak-gil, Chuncheon-si, Gangwon-do 24252, Korea \\ ${ }^{6}$ Biomedux Co., Ltd., \\ 4th Avenue 17 Ace Gwanggyo Tower 1 308, Yeongtong-gu, Suwon-si, Gyeonggi-do 16226, Korea
}

(Received April 5, 2018; accepted March 20, 2019)

Keywords: electrical cell-substrate impedance spectroscopy, analog front end, impedance, web-based graphical user interface

We propose an electrical cell-substrate impedance sensing (ECIS) system consisting of a low-cost compact analog front end (AFE) and a web-based user interface. Adopting a latemodel oscilloscope reduces the cost and size of the AFE. The web server was installed on an embedded computer to control the AFE and user interactions. The proposed system frees up the space normally needed for a display and keyboard/mouse in existing systems, which is an advantage in crowded laboratories, and it also prevents malfunctions due to inadvertent user input. In addition, the system control and experimental status can be monitored via smart devices such as smartphones and tablets anytime and anywhere. The accuracy and precision of the impedance measurement, which is the basic function of the ECIS system, are verified through experiments. Biomedical engineers using the proposed system report benefits due to the saving of laboratory space, the reduced probability of malfunction, the potential to monitor the experiment anytime and anywhere, and the ability to share experimental results with remote colleagues.

\section{Introduction}

Electrical cell-substrate impedance sensing (ECIS) technology can be applied to the analysis of cell migration, attachment and spreading, transepithelial electrical resistance, and proliferation. ${ }^{(1-10)}$ An electrical cell-substrate impedance sensor is one of the electrical biosensors that perform biochemical analysis entirely dependent on current and/or voltage

*Corresponding author: e-mail: jongwonkim33@gmail.com https://doi.org/10.18494/SAM.2019.2282 
measurements. ${ }^{(11,12)}$ Using the impedance sensor, we can observe the cytotoxicity of a variety of toxicants and the status of stem and cancer cells. ${ }^{(13-19)}$ When cells are added to the electrical cell-substrate impedance sensor array and attached to the electrodes, they act as insulators that increase the impedance. As the cells grow and cover the electrodes, the number, shape, and attachment properties of these cells affect the current flow and change the impedance. Observations of changes in impedance over time can be used to inversely predict the cell status.

Conventionally, a lock-in amplifier is used to measure the impedance of a cell substrate, but a signal acquisition system such as a digital oscilloscope and a sine wave generator can be used to measure the impedance. ${ }^{(20)}$ Although a precision impedance analyzer is usually used in research, application-specific integrated chips can also be used for portability or high throughput. ${ }^{(21-23)}$ Since most applications require the measurement of impedance below $100 \mathrm{kHz}$, the signal generation and acquisition must be stable in this range. The ECIS system is used to observe impedance changes over time, so it is essential to graphically display the experimental data. This can be accomplished using a dedicated ECIS system or a data processing and visualization application. ${ }^{(8,9,17,24,25)}$ For portable devices, a personal computer (PC) may be connected to the impedance measuring device. ${ }^{(21)}$ The control of the graphical user interface (GUI) requires input devices such as a mouse and keyboard as well as a display device such as an LCD monitor. However, the input and display devices not only increase the overall size of the system, but also increase the likelihood of inadvertent user interaction. This problem is more significant when the ECIS system is implemented in the form of point-of-care systems, which are becoming increasingly common today. Since ECIS instruments measure the impedance signal every few minutes over a period of several days, the user does not need to check the results continually. Thus, if the user interface is separated from the impedance measurement device, it can both save space and reduce the risk of improper user interaction with the device.

The number of biomedical devices with GUIs has increased over the past decades as GUIs are popular and provide more convenient user interaction. Generally, a PC with the Windows operating system or its embedded version provides a powerful GUI development environment and excellent maintenance functions. ${ }^{(26-28)}$ However, it is anticipated that rapid and recent developments in open hardware and software platforms will be actively applied to developing GUIs. ${ }^{(29-31)}$ Regardless of the platform, the GUI is typically a desktop application; however, with the development of Internet-of-Things and cloud computing, the display and data records associated with biomedical equipment are expected to become cloud-based to facilitate maintenance, control, and monitoring. With these changes, GUI implementation on the web is also expected. ${ }^{(29,32,33)}$ Implementing the GUI of a biomedical device on the web will help eliminate inadvertent keyboard input by removing the separate input devices. In addition, the space required for input/output devices is greatly reduced, providing users with more space.

In this paper, we describe a system in which the space occupied by the ECIS system is reduced, which prevents inadvertent GUI interaction by introducing a low-cost, compact design of the analog front end (AFE) and a web-based user interface. To implement this compact AFE, a USB oscilloscope with a signal generation function was adopted and the impedance was measured by controlling it via an embedded computer. The control of the measurement program 
and the display of the results are performed via a web interface for smart devices connected to the Internet. This allows the space occupied in the laboratory to be drastically reduced, and the impedance change can be observed anytime and anywhere. The performance of the proposed system was verified experimentally using an emulator circuit board for an electrical cell-substrate impedance sensor array and actual cell culture experiments. Experimental results show that the proposed system works well. In addition, we received very positive responses from bioengineers who conducted cell culture experiments for several months using the proposed system. The following section details the implementation of the AFE and web server, how to measure the impedance, and the cell-culture experimental process. Sections 3 and 4 describe the experimental results and conclusions, respectively.

\section{Materials and Methods}

\subsection{Overall system implementation}

Figure 1 shows the block diagrams of the proposed system and a classical system employing a PC GUI. Existing systems implement a desktop application that provides a GUI on a PC to visualize the impedance data obtained by controlling the AFE. In contrast, our proposed system implements a web server on an embedded computer that allows the AFE data to be collected and visualized by instructions received from smart devices. With this structure, a small embedded computer without a display or input devices can be used for the web server. In addition, only the web server and AFE are installed in the laboratory, reducing the footprint of the system and preventing inadvertent input.

\subsection{AFE implementation}

Figure 2 shows the sensor array interface circuitry. For noninvasive detection, the stimulus current should be about $1 \mu \mathrm{A}$ and the resistance of the cell-loaded channel is on the order of

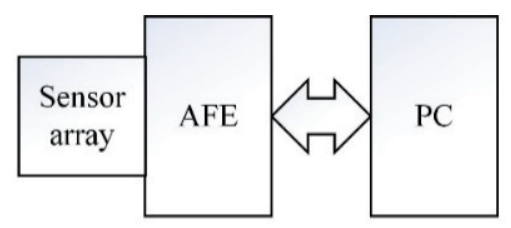

(a) classical system

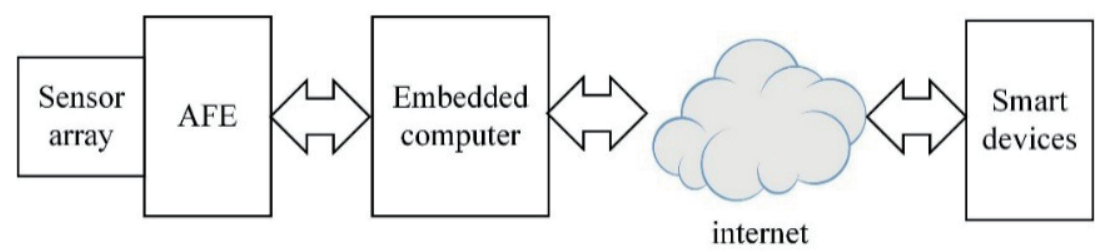

(b) proposed system

Fig. 1. (Color online) Overall system. 


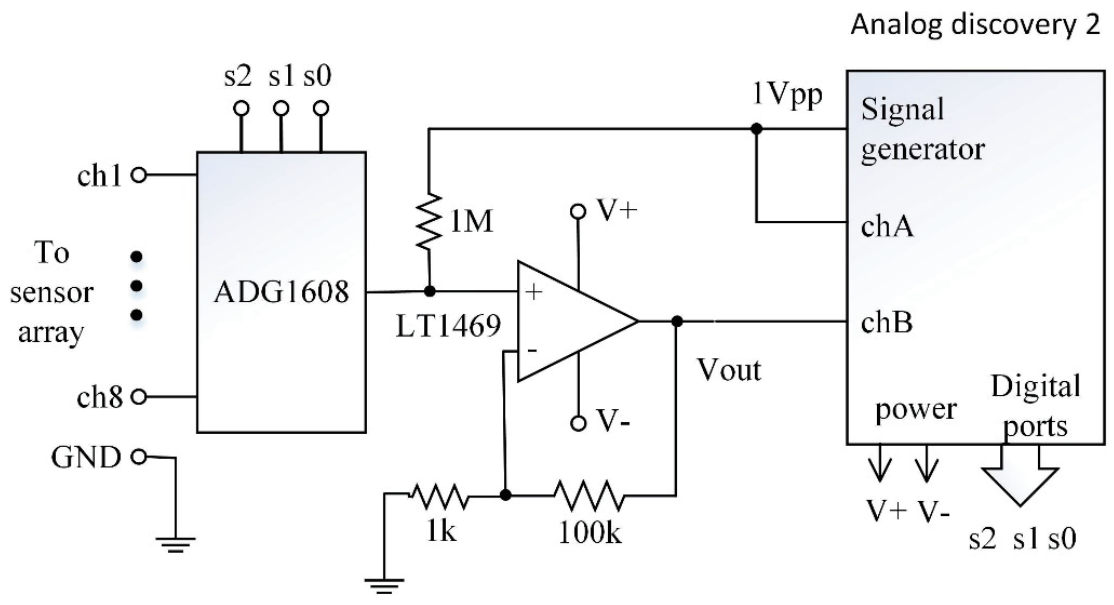

Fig. 2. AFE block diagram.

$10 \mathrm{k} \Omega$. Thus, as shown, the $1 \mathrm{Vpp}$ signal is connected to the multiplexer through a $1 \mathrm{M} \Omega$ resistor. When this circuit is configured, the selected channel through the multiplexer is stimulated with a sine wave current of about $1 \mu \mathrm{A}$. The specifications for the amplifier type and gain were determined through a review of the literature. In most studies, the reported frequency of the stimulation current was within $100 \mathrm{kHz}$ and the channel resistance of the cell was within $50 \mathrm{k} \Omega$. The gains were less than 100 considering the saturation of the amplifier output. As a result, 100 was the most appropriate gain, and we chose an amplifier with a gain bandwidth above $10 \mathrm{MHz}$ and low input offset current and voltage, considering the determined gain and a very low excitation current of less than $1 \mu \mathrm{A}$.

Considering performance, size, and cost, we chose a commercial USB oscilloscope (Analog Discovery 2, Digilent Inc., USA) as the signal generation and acquisition system. The device can generate a stable sine wave at $100 \mathrm{kHz}$ and provides a sufficiently large acquisition bandwidth. Since this equipment also provided the power and a digital port in addition to signal generation and two-channel signal acquisition, the amplifier power and multiplexer selection signals were also implemented through this equipment. The control of the USB oscilloscope was handled through web server software. The signals from the signal generator and the amplifier output were connected to channels A and B of the oscilloscope, respectively. Various methods could be adopted to obtain the impedance from these two signals. However, in this work, the sine functions of the two signals were estimated by a least-squares-error fitting method. The complex gain between the excitation voltage measured from channel $\mathrm{A}$ and the divided voltage measured at channel $\mathrm{B}$ could be calculated from the amplitude ratio and the phase difference between the matched sine functions. The relative impedance for the reference resistor (1 $\mathrm{M} \Omega$ resistor in Fig. 2) was related to the gain calculated as

$$
g=\frac{Z}{Z_{r e f}+Z}
$$


where $g, Z$, and $Z_{\text {ref }}$ are the complex gain, the channel impedance to be measured, and the reference resistance $(1 \mathrm{M} \Omega)$, respectively. From the above equation, the impedance of each channel of the sensor array could be obtained.

Figure 3 shows the components of the implemented AFE. An array interface board containing a multiplexer and an amplifier circuit can be mounted on the Analog Discovery 2 oscilloscope, as shown on the left in the figure. The right side shows the array emulator board and sensor array (Applied BioPhysics, Inc., NY USA). At the bottom of the array interface board, there are nine spring contacts that are connected to the contact part of the sensor array by pressure.

Because the entire AFE must be in an incubator, it is sealed in a case, as shown in Fig. 4. When the lever shown in the middle of the figure is pushed in the direction of the arrow, the array contacts and the spring contacts of the array interface board become tightly connected and the inside of the AFE is sealed off from the incubator air. A flat USB cable was used to ensure that incubator sealing was disturbed as little as possible as it exited the incubator.

Figure 5 shows the system setup in which a mini PC (Zbox PI330, Zotac, China) was used as the embedded computer installed on top of the incubator. The AFE, installed on the top shelf

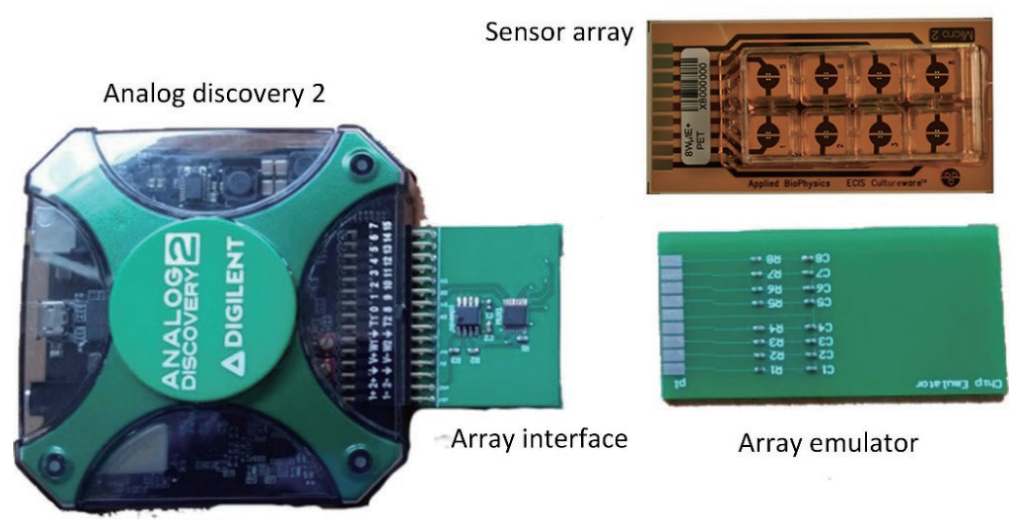

Fig. 3. (Color online) Components of the AFE.

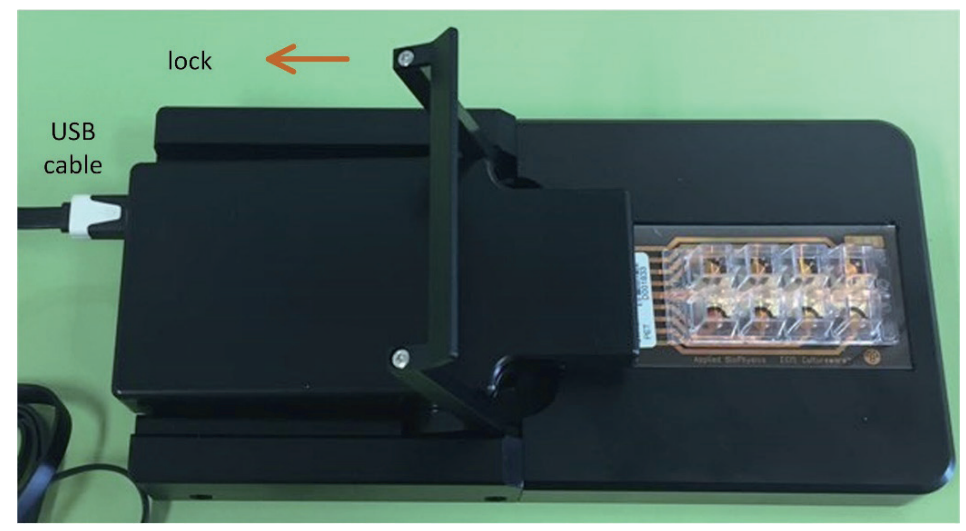

Fig. 4. (Color online) Sealed AFE. 


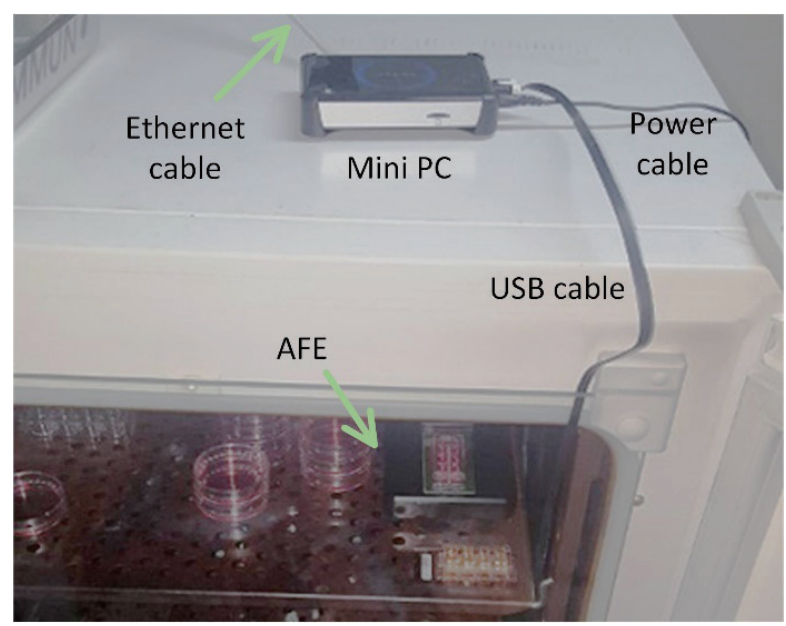

Fig. 5. (Color online) System setup.

inside the incubator, is connected by a USB cable. In addition to the USB cable, the mini PC is connected to a power cable and an ethernet cable.

\subsection{Emulator circuit board for the verification of impedance measurement performance}

To verify the impedance measurement performance of the proposed system, we fabricated a circuit board capable of emulating a sensor array, as shown in Fig. 6. Eight channels were each connected in series to a resistor with resistance increasing successively from 1 to $8 \mathrm{k} \Omega$ resistors and a $10 \mathrm{nF}$ capacitor to emulate the different degrees of cell proliferation. The impedance of each channel was repeatedly measured using the proposed system, and the coefficient of variation $(\mathrm{CV})$ was obtained. The relative error was also obtained by calculating the difference from the value measured with a digital multimeter.

\subsection{Software implementation}

Figure 7 shows a software block diagram of the proposed system. The ECIS system software can be divided into a device control module that controls the AFE, a web front-end module that manages the user interface, and a web server that controls these modules and manages the measurement data in the database. The selected software suite was demonstrated to work well with a variety of mini or embedded PCs running on the Windows operating system and with a Linux single-board computer. All modules were implemented in Python and could be run on all platforms without modification. The web server was designed with Python's Django Web Framework, an easy and powerful language. The web server displays the web front end to the user with a GUI, receives commands from the user, controls the device, and enables the visualization of the experimental data. 


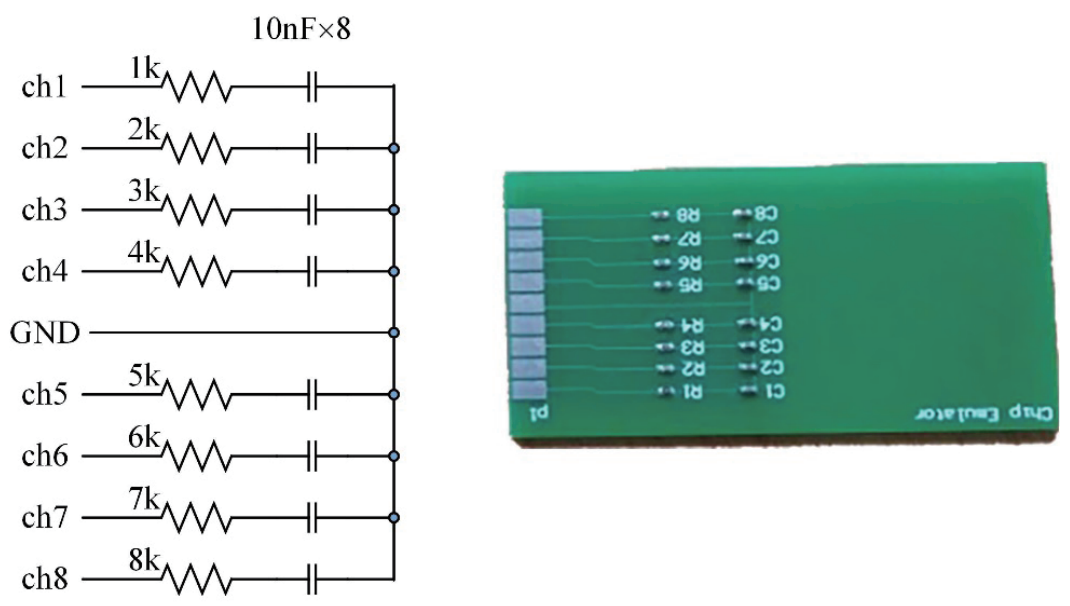

Fig. 6. (Color online) Sensor array emulator.

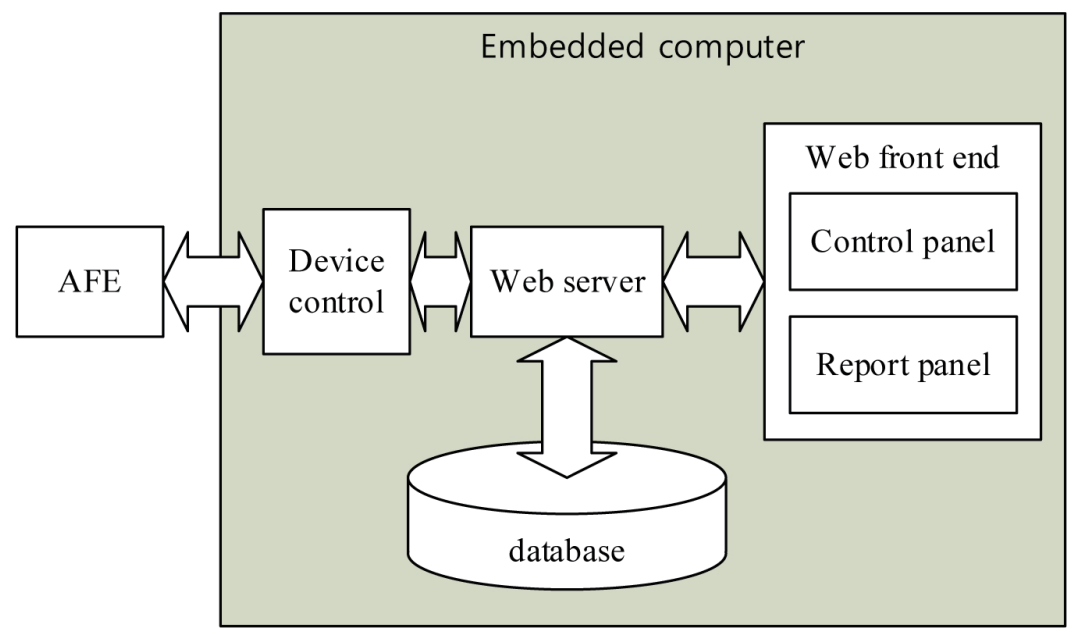

Fig. 7. (Color online) Overall software structure of the proposed ECIS system.

Figures 8 and 9 show the control panel for controlling the AFE and the report panel for confirming the experimental data, respectively. From the left, the control panel is divided into three parts: 'CHIP SETUP', 'PARAMETER SETUP', and 'DEVICE CONTROL'. When the user presses the 'CHECK CHIP' button located in the 'CHIP SETUP' section, the device measures the impedances from all the channels of the sensor array, outputs them to the table over the button, and activates 'PARAMETER SETUP'. If a channel is not loaded with a cell and the channel impedance exceeds the given threshold, instead of the ' $V$ ' icon, a red ' $x$ ' icon is displayed to indicate that the cell is absent, and the corresponding check box is disabled. Users can select the checkbox in the 'PARAMETER SETUP' section to set the desired channels and frequencies. The measurement interval and deadline can be arbitrarily set in units of minutes and days, respectively. After completing the desired experimental conditions in 'PARAMETER 


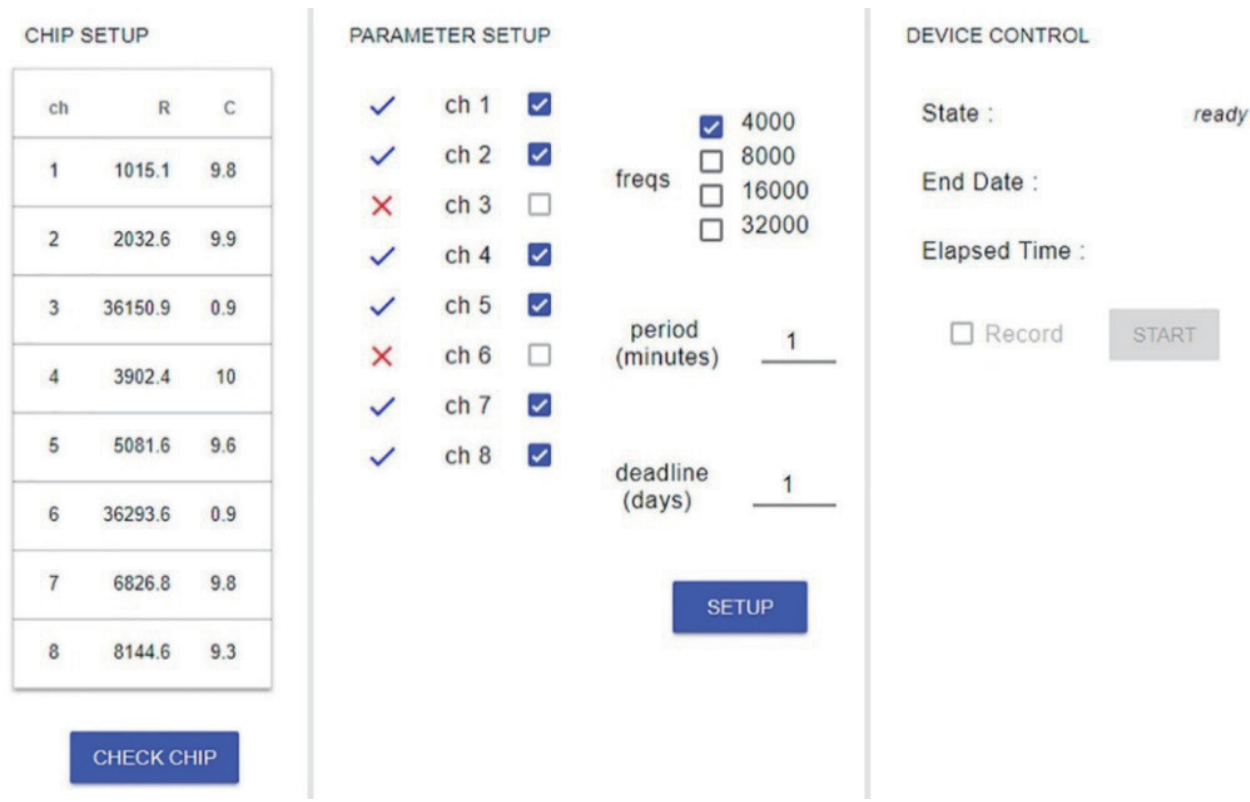

Fig. 8. (Color online) Control panel.

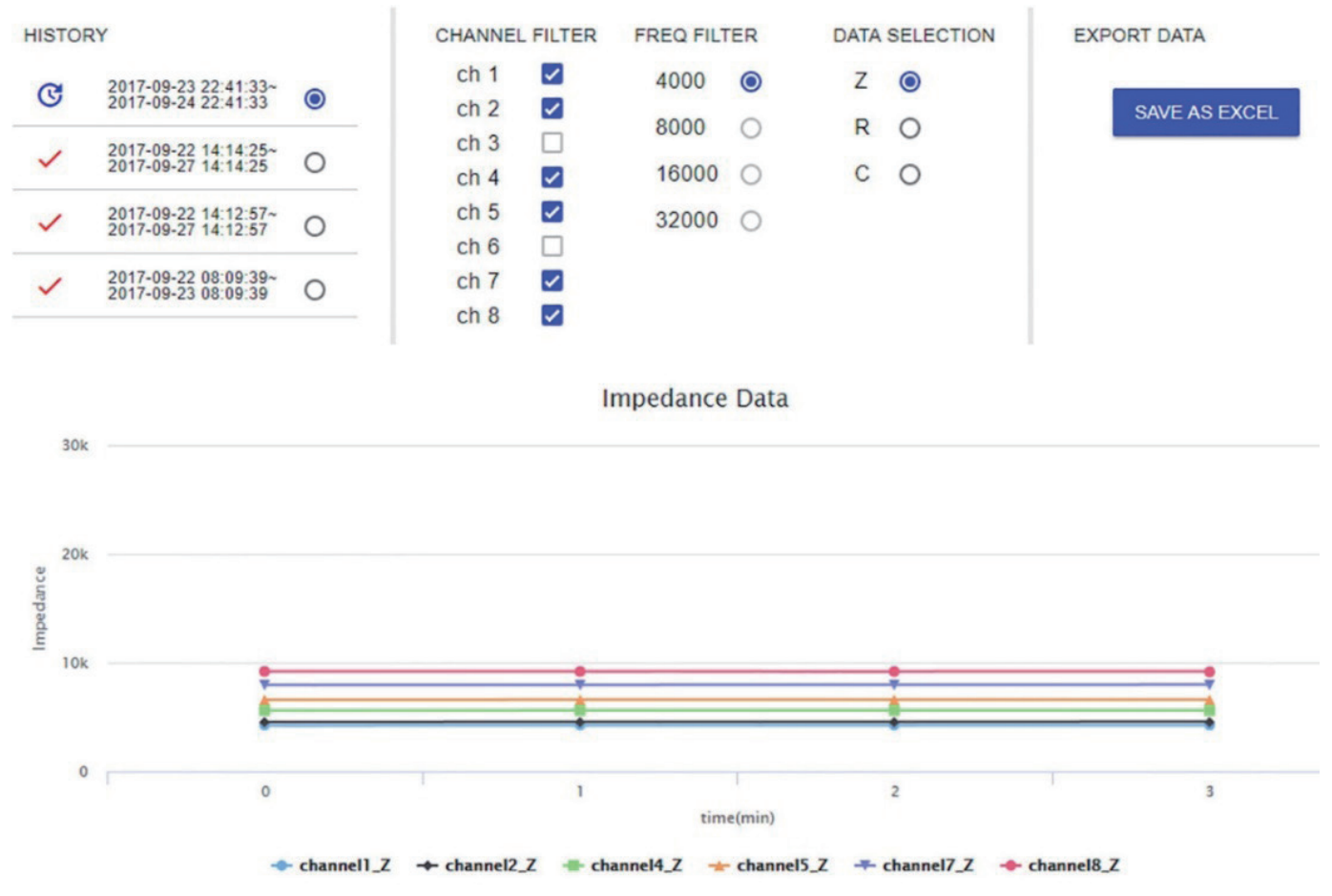

Fig. 9. (Color online) Report panel.

SETUP', the 'SET UP' button can be pressed to activate 'DEVICE CONTROL'. In this part, the status information of the current experiment is displayed as either 'initializing', 'ready', 'setup ok', or 'running' on the row labeled 'State:'. If the experiment is in progress, the deadline and elapsed time of the experiment are displayed on the rows labeled 'End Date:' and 'Elapsed 
Time:', respectively. The 'START' button can be pressed to start the measurement. The button labeled 'Record' can be checked at any time to record experimental data.

Figure 9 shows the report panel, which presents the measurement data recorded in the local database of the embedded computer. Among the experiments that have been carried out so far, the latest three experimental data points can be drawn by checking one of the radio buttons. There are options to draw multiple desired channels, to select a frequency, or to draw a complex impedance by selecting the magnitude, resistance, or capacitance. The report panel also provides a 'SAVE AS EXCEL' button to download the data to an Excel file if desired. The lower graph of the report panel displays the results of the emulator measured at 1 min intervals. Figures 8 and 9 show the results of the emulator with the resistors of channels 3 and 6 removed to emulate empty channels. It can be seen that the corresponding channels in the 'PARAMETER SETUP' part of the control panel are indicated by red ' $x$ ' icons, and that the corresponding check boxes in the middle of the report panel are deactivated.

\subsection{Cell culture and growth measurements}

To test the impedance changes resulting from cell proliferation, human embryonic kidney 293T (HEK-293T) cells were cultured in Dulbecco's Modified Eagle Medium (DMEM) (Lonza, USA) supplemented with 10\% fetal bovine serum (FBS) (Gendepot, USA) and 1X antibioticsantimycotics (Gibco, USA), and they were maintained in $5 \% \mathrm{CO}_{2}$ at $37^{\circ} \mathrm{C}$.

HEK-293T cells were cultured on an eight-well sensor array (8W1E PET, Applied BioPhysics, NY, USA), where the bottom surface of each well was coated with $0.01 \%$ poly-Llysine (Sigma Aldrich, USA) overnight at $4{ }^{\circ} \mathrm{C} . \quad 0.5-2 \times 10^{5}$ cells were plated in each well, and then their attachment was confirmed with a phase contrast microscope (JuliTMBr, NanoEntek, Korea) before being placed in the AFE. The sensor array has a circular electrode of $250 \mu \mathrm{m}$ at the center of each well such that the impedance data could be obtained through this electrode. As the cells grow and cover more of the electrode, the channel impedance increases.

\section{Results and Discussion}

Table 1 shows the CVs for the repeated measurements of various amplifier gains and stimulus frequencies. All measurements were taken for the $1 \mathrm{k} \Omega$ channel of the array emulator. The reason for selecting the $1 \mathrm{k} \Omega$ channel was that the deviation was the largest when the divided voltage was the smallest. Each $\mathrm{CV}$ was calculated by dividing the standard deviation of the five measurements for each gain and each frequency by the average. It was confirmed that

Table 1

Coefficients of variation for five repeated measurements using the proposed system ( $1 \mathrm{k} \Omega$ channel, \%).

\begin{tabular}{cccccccccc}
\hline \multirow{2}{*}{ Amp. } & \multicolumn{8}{c}{ Frequency $(\mathrm{kHz})$} \\
\cline { 2 - 9 } & 1 & 2 & 4 & 8 & 16 & 43 & 64 & 128 & Mean \\
\hline 10 & 0.9 & 0.5 & 0.3 & 0.4 & 0.5 & 0.4 & 0.9 & 0.6 & 0.6 \\
50 & 0.2 & 0.1 & 0.0 & 0.2 & 0.1 & 0.3 & 0.1 & 0.4 & 0.2 \\
100 & 0.1 & 0.1 & 0.1 & 0.1 & 0.2 & 0.3 & 0.3 & 0.2 & 0.2 \\
\hline
\end{tabular}


the $\mathrm{CV}$ was within $0.6 \%$ regardless of gain or frequency. When the gain was fixed at 50 and 100 , the performance was almost the same. However, since the channel impedance could be less than $1 \mathrm{k} \Omega$, the gain was fixed at 100 when fabricating the array interface board.

Table 2 shows the relative errors of resistance and capacitance measured using the proposed system. The resistance was compared with the value measured with a digital multimeter and the capacitance was assumed to be $9.5 \mathrm{nF}$. Overall, the resistance was within 3.6\%. As expected, the smaller the resistance, the greater the relative error. The assumed and meaured spray capacitances from the array interface board to the emulator wiring would have affected the error, but the overall error is very small. Considering that the relative error and measurement variation are very small, as shown in Tables 1 and 2, the proposed system can be used without correction in most applications. For more precise applications, a calibration would be effective because the measurement variations are small, as shown in Table 1.

Figure 10 shows a microscopy image showing the cell attached to one well of the sensor array $8 \mathrm{~W} 1 \mathrm{E}$. The bright circle on the right shows the working electrode, and the more cells that cover the electrode, the higher the impedance. Figure 11 shows that the impedance increases with cell proliferation. The blue curve corresponds to the case with the most cells loaded.

In addition to easily observing the cell proliferation status with the proposed system, bioresearchers have reported satisfaction with the following features. First, the proposed system does not occupy much space in the laboratory, and there is no concern that an accessible input device such as a keyboard or a mouse would lead to inadvertent input malfunction. Second, an

Table 2

Relative errors of the proposed system (4 kHz, $1 \mathrm{Vpp}$, sine wave stimulation, \%).

\begin{tabular}{ccccccccc}
\hline & \multicolumn{8}{c}{ Channel } \\
\cline { 2 - 10 } & 1 & 2 & 3 & 4 & 5 & 6 & 7 & 8 \\
\hline$R$ & 3.6 & 1.7 & 1.2 & 0.9 & 0.4 & 0.2 & 0.2 & 0.0 \\
$C$ & 1.4 & -2.5 & 1.3 & 1.5 & 2.6 & 0.5 & 0.3 & 0.6 \\
\hline
\end{tabular}

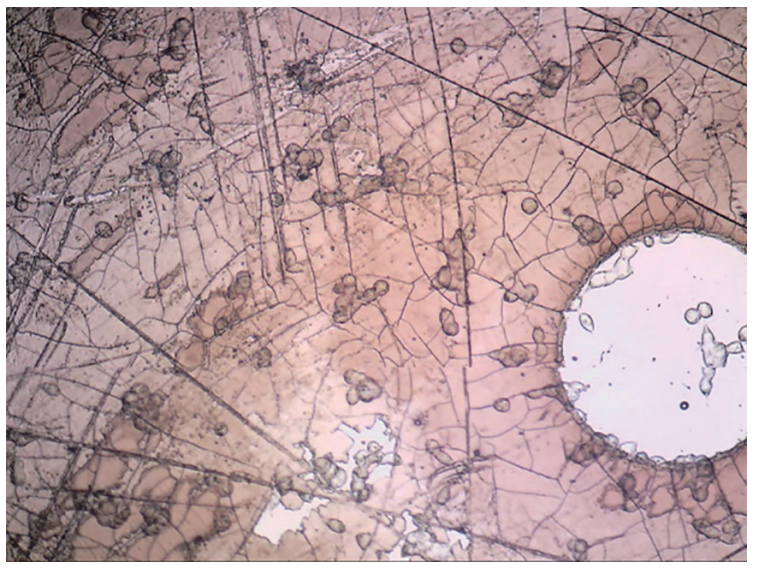

Fig. 10. (Color online) Channel with a loaded cell. 


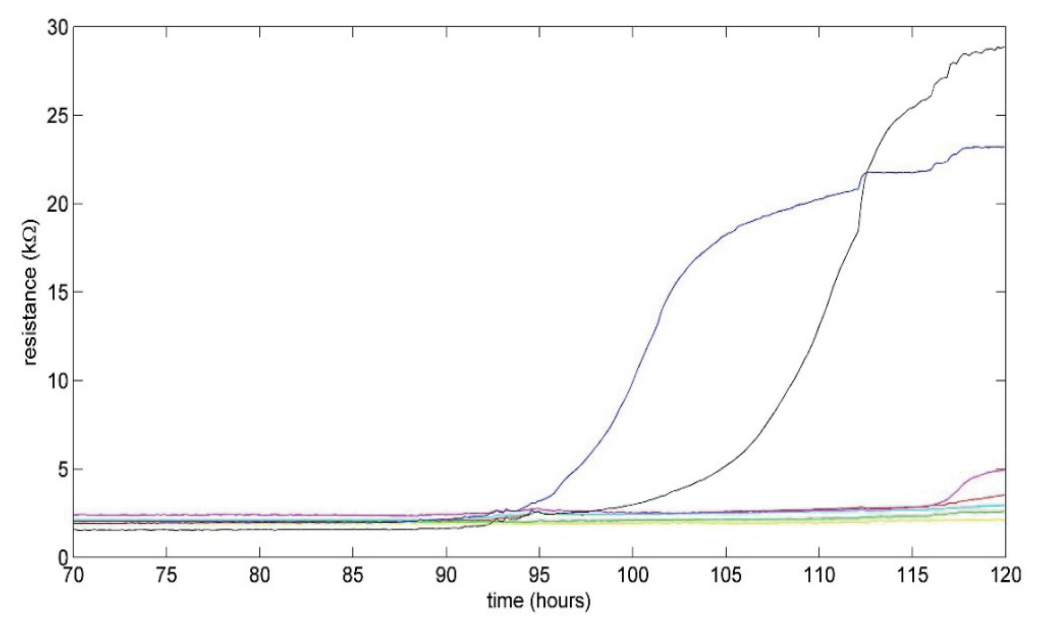

Fig. 11. (Color online) Impedance change.

Internet-enabled device such as a smartphone or a tablet enables access to the server anytime and anywhere without the need for an application program to control the system. The user interface shows the progress of the current experiment or results of a previous experiment. It is also possible to share the experiment with remote collaborators. Finally, any concern about data loss due to network failure is eliminated because the data is stored continuously and reliably in the local database.

\section{Conclusions}

We proposed an ECIS system with a reduced size of the AFE owing to the adoption of a small late-model oscilloscope and the utilization of a web server on the embedded computer to provide AFE control and a web-based user interface. The accuracy and precision of the impedance measurement, which is the basic function of the ECIS system, were verified through experiments. The proposed system can be used in complex laboratories because it saves space since there is no need for a display or a keyboard and mouse. In addition, smart devices such as smartphones and tablets enable flexible system control and the monitoring of the experiment status, as well as access anywhere with an Internet connection. Bioengineers using this equipment reported satisfaction with the proposed system since it saves laboratory space, reduces the probability of inadvertent input malfunction, enables experimental control anytime and anywhere, allows experiments to be shared with remote colleagues, and stores data stably and continuously to the local database.

\section{Acknowledgments}

This research was supported by the Basic Science Research Program through the National Research Foundation of Korea (NRF), funded by the Ministry of Science, ICT \& Future 
Planning (NRF-2017R1A2B4002679), and by The Leading Human Resource Training Program of Regional Neo industry through the National Research Foundation of Korea (NRF), funded by the Ministry of Science, ICT \& Future Planning (NRF-2016H1D5A1909654).

\section{References}

1 X. Zhang, W. Wang, A. N. Nordin, F. Li, S. Jang, and I. Voiculescu: Sens. Actuator, B 247 (2017) 780.

2 J. Hong, M. Chu, L. Qian, J. Wang, Y. Guo, and D. Xu: BioMed Res. Int. 2017 (2017) 1.

3 J. Wegener, C. R. Keese, and I. Giaever: Exp. Cell Res. 259 (2000) 158.

4 I. Giaever and C. R. Keese: Proc. Natl. Acad. Sci. U.S.A. 88 (1991) 7896.

5 I. Giaever and C. R. Keese: Nature 366 (1993) 591.

6 H. Gong, M. Liu, J. Klomp, B. J. Merrill, J. Rehman, and A. B. Malik: Sci. Rep. 7 (2017) 42127.

7 Y. Cui, Y. An, T. Jin, F. Zhang, and P. He: Sens. Actuator, B 250 (2017) 461.

8 B. A. Nanes, C. M. Grimsley-Myers, C. M. Cadwell, B. S. Robinson, A. M. Lowery, P. A. Vincent, M. Mosunjac, K. Fruh, and A. P. Kowalczyk: Mol. Biol. Cell 28 (2016) 30.

9 J. P. Garrett, A. M. Lowery, A. Adam, A. P. Kowalczyk, and P. A. Vincent: Mol. Biol. Cell 28 (2016) 85.

10 A. P. Adam, A. M. Lowery, N. Martino, H. Alsaffar, and P. A. Vincent: PLoS ONE 11 (2016) 1.

11 I.-F. Cheng, T.-Y. Chen, and W.-C. Su: Int. J. Eng. Technol. Innovation 6 (2016) 225.

12 J. S. Daniels and N. Pourmand: Electroanalysis 19 (2007) 1239.

13 A. Bartlett, A. J. Sanders, F. Ruge, K. G. Harding, and W. G. Jiang: Exp. Therapeutic Med. 12 (2016) 33.

14 A. Wagner, R. Eldawud, A. White, S. Agarwal, T. A. Stueckle, K. A. Sierros, Y. Rojanasakul, R. K. Gupta, and C. Z. Dinu: Biochim. Biophys. Acta 1861 (2017) 3406.

15 R. C. Nordberg, J. Zhang, E. H. Griffith, M. W. Frank, B. Starly, and E. G. Loboa: Stem Cell Transl. Med. 6 (2017) 502.

16 S. E. Kispert, J. Marentette, E. C. Campian, T. S. Isbell, H. Kuenzel, and J. McHowat: Physiol. Rep. 5 (2017) e13177.

17 A. Elfick, G. Rischitor, R. Mouras, A. Azfer, L. Lungaro, M. Uhlarz, T. Herrmannsdörfer, J. Lucocq, W. Gamal, P. Bagnaninchi, S. Semple, and D. M. Salter: Sci. Rep. 7 (2017) 39755.

18 Y. Zhou, S. Basu, E. Laue, and A. A. Seshia: Biosens. Bioelectron. 81 (2016) 249.

19 T. B. Tran, C. Baek, and J. Min: PLoS One 11 (2016) e0153813.

20 H. Ma, R. W. Wallbank, R. Chaji, J. Li, Y. Suzuki, C. Jiggins, and A. Nathan: Sci. Rep. 3 (2013).

21 K. Chabowski, T. Piasecki, A. Dzierka, and K. Nitsch: Metrol. Meas. Syst. 22 (2015) 13.

22 C. J. Chen, J. T. Liu, S. J. Chang, M. W. Lee, and J. Z. Tsai: J. Taiwan Inst. Chem. E 43 (2012) 678.

23 T. Schwarzenberger, P. Wolf, M. Brischwein, R. Kleinhans, F. Demmel, A. Lechner, B. Becker, and B. Wolf: Physiol. Meas. 32 (2011) 977.

24 K. R. Schiller, P. J. Maniak, and S. M. O’Grady: Am. J. Physiol. Cell Physiol. 299 (2010) C912.

25 T. B. Tran, P. D. Nguyen, C. Baek, and J. Min: Biosens. Bioelectron. 77 (2016) 631.

26 W. Huang, F. Fang, X. Ma, Z. Dong, and X. Xu: Industrial Electronics and Applications (ICIEA), 2015 IEEE 10th Conf. (2015) 2030.

27 M. Qi, G. Cheng, Y.-L. Xu, and K. Li: 2014 Int. Conf. Automatic Control Theory and Application (ACTA-14) (2014).

28 P. K. Bhagwat and H. Zodpe: Embedded Systems (ICES), 2014 Int. Conf. (2014) 157.

29 A. Ahmad, A. Paul, M. M. Rathore, and H. Chang: FGCS NLM 56 (2016) 493.

30 A. Lewis, M. Campbell, and P. Stavroulakis: Measurement 87 (2016) 228.

31 G. Mashette, P. Borole, and S. Bhat: Recent Trends in Electronics, Information \& Communication Technology (RTEICT), IEEE Int. Conf. (2016) 1407.

32 A. Ukil, S. Bandyoapdhyay, C. Puri, and A. Pal: Advanced Information Networking and Applications (AINA), 2016 IEEE 30th Int. Conf. (2016) 994.

33 A. Botta, W. De Donato, V. Persico, and A. Pescapé: FGCS NLM 56 (2016) 684. 


\section{About the Authors}
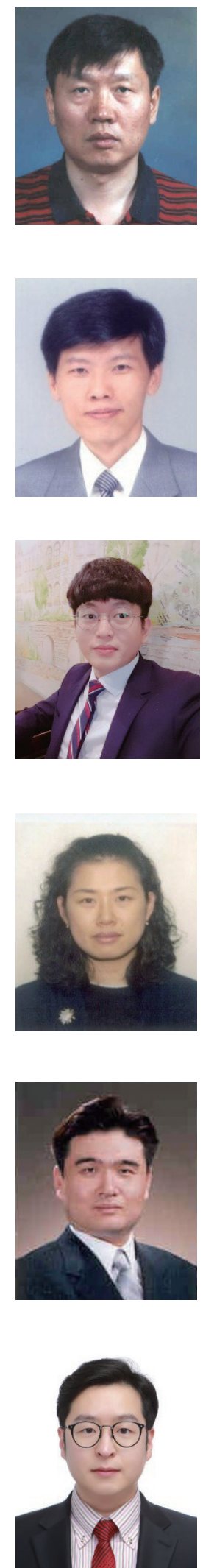

Jong-Dae Kim received his M.S. and the Ph.D. degrees in electrical and electronics engineering from Korea Advanced Institute of Science and Technology, Seoul, Korea, in 1984 and 1990, respectively. He worked for Samsung Electronics from 1988 to 2000 as an electronics engineer. He is currently a professor in the School of Software at Hallym University. His recent interests are focused on biomedical systems and bioinformatics.

Chan-Young Park received his B.S. degree from Seoul National University and his M.S. and Ph.D. degrees from Korea Advanced Institute of Science and Technology in 1989 and 1995, respectively. From 1991 to 1999, he worked at Samsung Electronics. He is currenlty a professor in the School of Software at Hallym University. His research interests are in the areas of Bio-IT convergence, intelligent transportation systems, and sensors.

Sang-Oh Park received his B.S. degree in computer engineering from Hallym University. He is currently working for DOUZONE ICT GROUP, Korea, as an IT engineer. His research interests include IoT systems and cloud technology.

Hye-Jeong Song received her Ph.D. degree in computer engineering from Hallym University. She is currently a research professor at the Bio-IT Research Center at Hallym University. Her recent research is in the areas of biomedical systems and bioinformatics.

Yu-Seop Kim received his Ph.D. degree in computer engineering from Seoul National University. He is currenlty a professor in the School of Software at Hallym University. His research interests are in the areas of bioinformatics, computational intelligence, and natural language processing.

Won-Kyun Im received his M.S. degree from Hallym University. He is currently pursuing a Ph.D. degree in the Department of Life Science at Hallym University. He is a researcher at the Korea National Cancer Center. His research interests include molecular immunology and cell-based functional analysis. 


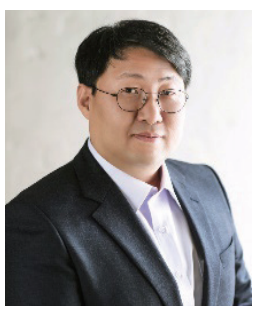

Sung-Ho Jeon received his Ph.D. degree in molecular biology from Seoul National University. He is currently a professor in the Department of Life Science at Hallym University. His recent research is in the areas of molecular immunology and cell-based functional analysis.

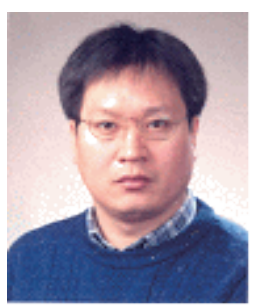

Jongwon Kim received his B.S. and M.S. degrees in physics from Seoul National University in 1985 and 1987, respectively. He received his Ph.D. degree in biomedical engineering from Soul National University in 1992. He has been the director of the Medical Development Center at Daegu Gyeongbuk Advanced Medical Industry Promotion Foundation since 2018. His research interests include biomedical systems and bioinformatics. 